\title{
Synthesis of Fe Nanoparticles Functionalized with Oleic Acid Synthesized by Inert Gas Condensation
}

\author{
L. G. Silva, ${ }^{1}$ F. Solis-Pomar, ${ }^{1}$ C. D. Gutiérrez-Lazos, ${ }^{1}$ Manuel F. Meléndrez, ${ }^{2}$ E. Martinez, ${ }^{3}$ \\ A. Fundora, ${ }^{4}$ and E. Pérez-Tijerina ${ }^{1}$ \\ ${ }^{1}$ Facultad de Ciencias Físico-Matemáticas, Universidad Autónoma de Nuevo León, 66451 San Nicolás de los Garza, NL, Mexico \\ ${ }^{2}$ Hybrid Materials Laboratory (HML), Department of Materials Engineering (DIMAT), Faculty of Engineering, \\ University of Concepcion, 270 EdmundoLarenas, Casilla 160-C, 4070409 Concepcion, Chile \\ ${ }^{3}$ Centro de Investigación en Materiales Avanzados S. C., Unidad Monterrey-PIIT, 66600 Apodaca, NL, Mexico \\ ${ }^{4}$ Instituto de Ciencia y Tecnología de Materiales (IMRE), Universidad de La Habana, Zapata y G, Vedado, CP 10400 La Habana, Cuba
}

Correspondence should be addressed to E. Pérez-Tijerina; eduardo.pereztj@uanl.edu.mx

Received 17 March 2014; Revised 30 July 2014; Accepted 30 July 2014; Published 18 September 2014

Academic Editor: Renyun Zhang

Copyright (C) 2014 L. G. Silva et al. This is an open access article distributed under the Creative Commons Attribution License, which permits unrestricted use, distribution, and reproduction in any medium, provided the original work is properly cited.

In this work, we study the synthesis of monodispersed Fe nanoparticles (Fe-NPs) in situ functionalized with oleic acid. The nanoparticles were self-assembled by inert gas condensation (IGC) technique by using magnetron-sputtering process. Structural characterization of Fe-NPs was performed by transmission electron microscopy (TEM). Particle size control was carried out through the following parameters: (i) condensation zone length, (ii) magnetron power, and (iii) gas flow (Ar and He). Typically the nanoparticles generated by IGC showed diameters which ranged from $\sim 0.7$ to $20 \mathrm{~nm}$. Mass spectroscopy of Fe-NPs in the deposition system allowed the study of in situ nanoparticle formation, through a quadrupole mass filter (QMF) that one can use together with a mass filter. When the deposition system works without quadrupole mass filter, the particle diameter distribution is around $+/-20 \%$. When the quadrupole is in line, then the distribution can be reduced to around $+/-2 \%$.

\section{Introduction}

In the last decades, nanotechnology research has increased considerably. This is an interdisciplinary area which includes physics [1], chemistry [2], biochemistry [3], molecular biology [4], medicine [5], optoelectronic devises [6], and protein engineering [7], among others. The objectives of nanotechnology are the study, the understanding, and the control of the matter at atomic and molecular level. The investigation in nanotechnology has been centered principally in surface modified nanoparticles [8], advanced administration in medicaments [9], transistors [10], interfaces with biological entities [11], and other areas of the integration of nanosystems or nanoelectronics with biological entities as the oriented to the delivery of active biological entities [12] and; processing, manipulation, and detection of molecules or biological complexes [13]. Size control of the nanostructure allows obtaining new materials with functional and structural size-dependent properties. At this point, the research has focused basically on alloys and nanostructured composites [14], advanced functional polymeric materials [15], advanced functional nanostructured materials, and the incorporation of ordered molecular systems or nanoparticles in adequate substrates. However, the size control, shape, stability, and dispersibility of NPs in desired solvents are still a technological challenge. Therefore, providing proper surface coating and developing some effective protection strategies to keep the stability of NPs are very important. An unavoidable problem associated with particles in this size range is their intrinsic instability over longer periods of time. Such small particles tend to form agglomerates that reduce the energy associated with the high surface area to volume ratio of the nanosized particles. Moreover, naked metallic nanoparticles are chemically highly active and are easily oxidized in air, resulting generally in loss of magnetism and dispersibility, such as iron nanoparticles. For many applications it is crucial 


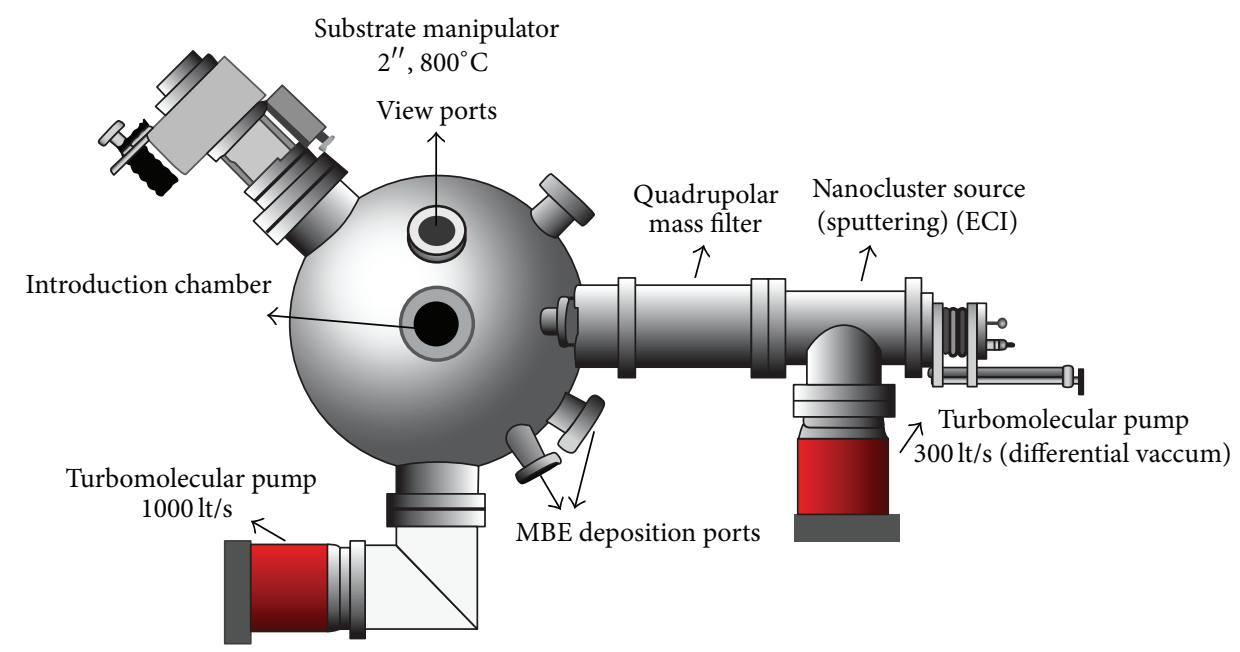

FIGURE 1: Integral system to synthesize Fe nanoparticles (image taken from Nanosys500 Manual [17]).

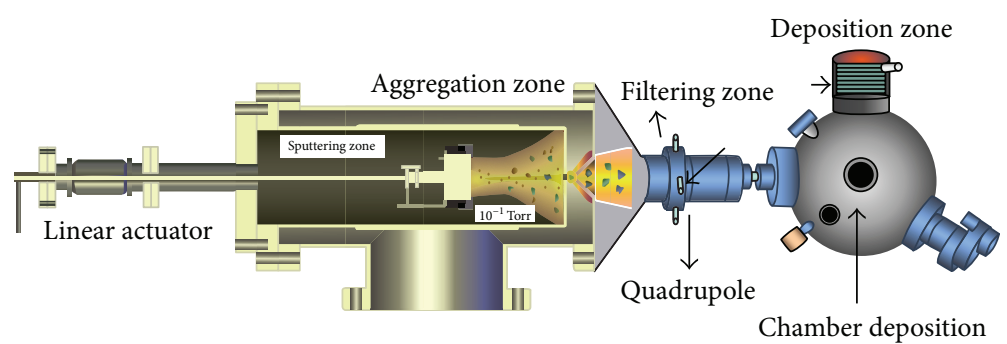

FIGURE 2: Schematic diagram of the nanocluster source.

to develop protection strategies to chemically stabilize the naked magnetic nanoparticles against degradation during or after the synthesis. These strategies comprise grafting of or coating with organic species, including surfactants or polymers, or coating with an inorganic layer, such as silica or carbon. It is noteworthy that in many cases the protecting shells not only stabilize the nanoparticles but also can be used for further functionalization, depending on the desired application [16].

A new generation of analysis instruments and fabrication at nanometric scale has been developed, for example, the system for the deposition and nanoparticle characterization from Mantis Deposition System (Nanosys500) [17] which allows obtaining nanoparticles with a controlled size ranging from 20 atoms up to $20 \mathrm{~nm}$. Chemical methods have as principal advantage their low cost, but they have as main deficiency their difficulty to obtain nanoparticles with low size dispersion. Only under strict conditions (inert atmospheres, short reflux times, and reaction temperatures) it is possible to obtain nanoparticles with a small deviation in their size [18]. Conversely, in a chemical synthesis it has nanoparticles with sizes ranging from $5 \mathrm{~nm}$ to $100 \mathrm{~nm}$, and this represents a technological disadvantage, considering that nanoparticle properties are mainly associated with their size. Another deficiency is the difficulty in growing nanoparticles with more than one component, for example, bimetallic nanoparticles, where it is necessary to have a precise and strict control of the chemical composition. On the contrary, the use of physical methods enables obtaining stoichiometric nanoparticles with controlled size and shape [14]. However, the high cost of these systems represents their main disadvantage.

This paper presents the results concerned with too structural and chemical characterization of Fe nanoparticles synthesized in the inert gas condensation system Mantis Nanosys500 and deposited on silicon substrates.

\section{Experimental Details}

Nanoparticles were prepared by the inert gas condensation (IGC) technique [19-21], using the Nanosys500 system (Mantis Deposition Ltd.) that employs sputtering technique as ion source [17]. The experimental set-up employed in this work for the production of size-selected Fe clusters is shown in Figure 1. In this figure, one can see the main parts of the system; it has a nanocluster source that includes a DC magnetron-sputtering unit and the turbo molecular pump $(300 \mathrm{~L} / \mathrm{s})$, a quadrupole mass filter, and a deposition chamber. The nanocluster source is the heart of the system to obtain the Fe nanoparticles; this has a device called Nanogen 50, where nanoclusters are produced and then they are channeled to the main deposition chamber to deposit them on the substrate as illustrated in Figure 2.

In the gas-phase condensation technique, a cathodic erosion system is used with a DC magnetron to generate the 
supersaturated metallic vapor. The size-selected Fe nanoparticles deposition takes place through four main processes: sputtering, aggregation, filtering, and deposition. In the gasphase condensation technique, a cathodic erosion system is used with a DC magnetron to generate the supersaturated metallic vapor. The size of the nanoparticles can be modified by three parameters: (i) condensation zone length (that can be varied from 30 to $150 \mathrm{~mm}$ ), (ii) magnetron power (ranging from 25 to $100 \mathrm{~W}$ ), and (iii) flow of gases (Ar and $\mathrm{He}$, partial pressure $1-2 \times 10^{-1}$ Torr). The condensation zone is defined as the distance between the magnetron head (erosion zone) and the first expansion aperture; this condensation zone can be modified by means of a linear drive actuator, reducing or increasing the distance between the magnetron head and the first expansion aperture, and in the same way, the accretion of the nanoparticle can be varied in time once the supersaturated vapor metal is aggregated, due to the increasing or decreasing of residence time for nanoparticles on the aggregation zone. Another parameter that modifies the nanoparticle size is the magnetron power; with higher magnetron power the sputtered atoms density from the target increases, leading to an increase of nanoparticle size. This relation has a linear increase as the saturation regime is reached, where an increment in power decreases slightly the nanoparticle size. The last parameter to modify the nanoparticle size is the gas flow ( $\mathrm{Ar}$ and $\mathrm{He}$ ), where $\mathrm{Ar}$ is used as erosion gas. By increasing the Ar flow the metal vapor pressure increases. When He gas was introduced in the chamber (which was used as carrier gas) and its flow was increasing, the NPs size is reduced; this reduction is associated with the collisions between the nanoparticles and the He molecules; thus the mean free path of nanoparticles is reduced, diminishing their size.

The Fe nanoparticles were deposited on Si substrates, with and without oleic acid covering in their surface. The Si substrates without oleic acid were sonicated in toluene to obtain a solution of Fe nanoparticles. The Si substrates with oleic acid were sonicated in toluene; the product was flocculated by adding ethanol and separated by centrifugation, decanting the supernatant. The precipitate was dispersed in toluene. The particles were characterized by transmission electron microscopy (TEM). In order to retain their crystalline characteristics and optical and electrical properties, the kinetic energy of nanoparticles was controlled by the energy cluster impact (ECI) technique $[22,23]$, and the energy of acceleration was kept at $0.1 \mathrm{eV}$ per atom in the nanoparticle, assuring a soft landing of nanoparticles on the substrate. In order to obtain monodispersed nanoparticles, the deposition time was few minutes up to 30 minutes.

\section{Results}

Among the advantages that Nanosys500 system can offer, we can mention, for example, that cluster distribution can be varied and the nanocluster source can be calibrated for optimum performance, in order to select the wished cluster size. The results of mass spectrum analysis for $1,3,4,5$, and $6 \mathrm{~nm}$ Fe nanoparticles are displayed in Figure 3. The

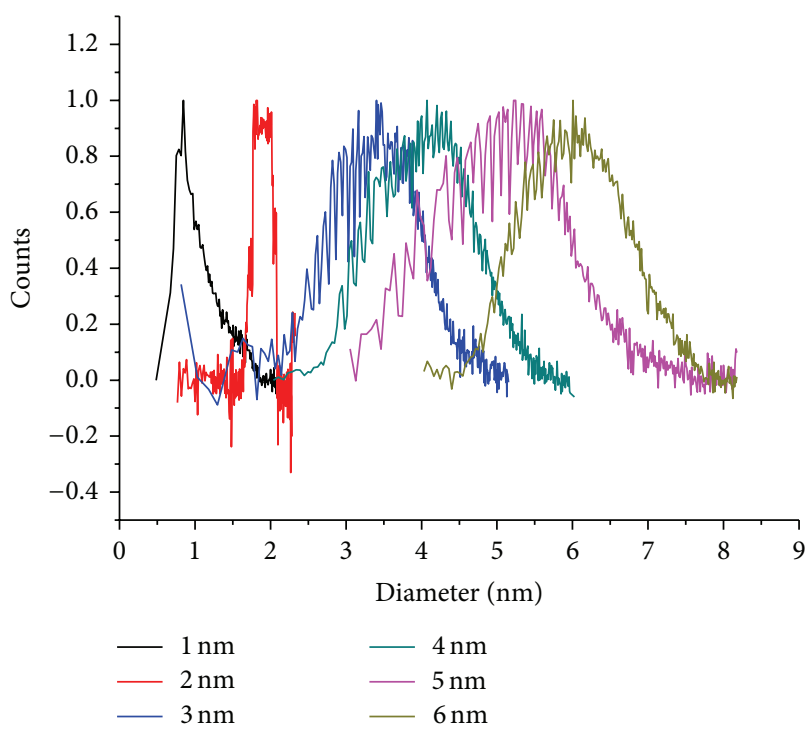

FIgURE 3: Mass spectrometry profiles for Fe nanoparticles of different sizes (before the selection by mass).

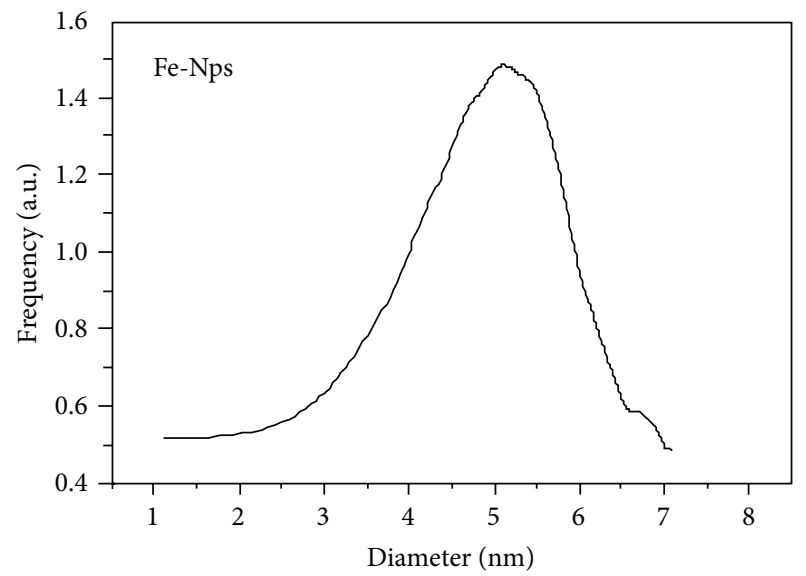

FIGURE 4: Mass spectra of filtered Fe-Nps $(5 \mathrm{~nm})$ obtained by IGC technique.

different mass profiles represent the capability of the inert gas condensation technique to prepare nanoparticles with a high resolved distribution size, and, hence, we can study their electrical, morphological, and optical properties based on the Fe nanoparticle size. Figure 4 displays the mass spectrum of Fe nanoparticles deposited on the Si substrates measured in the quadrupole mass spectrometer.

TEM image of filtered $5 \mathrm{~nm}$ nanoparticle was acquired to obtain the size distribution of deposited nanoparticles as shown in Figure 5(a). In this figure it is possible to distinguish a monodispersed size distribution of Fe nanoparticles centered at $5 \mathrm{~nm}$. Nanoparticles were filtered through the quadrupole mass spectrometer. A high magnification micrography is presented in Figure 5(b). The histogram in Figure 5(c) let us analyze the measured mean size, which demonstrates the narrow size dispersion in filtered nanoparticle size at $5 \mathrm{~nm}$ with a central value in $5.1 \mathrm{~nm}$, demonstrating 


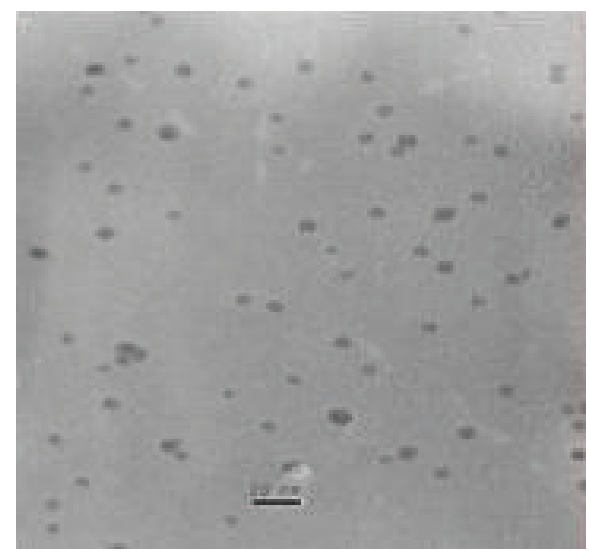

(a)

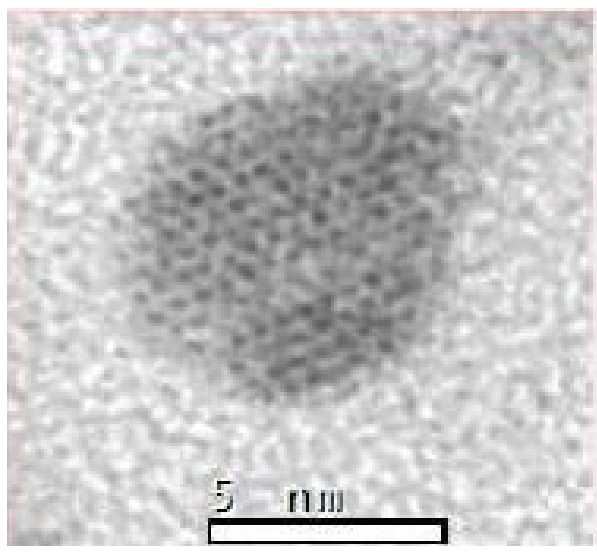

(b)

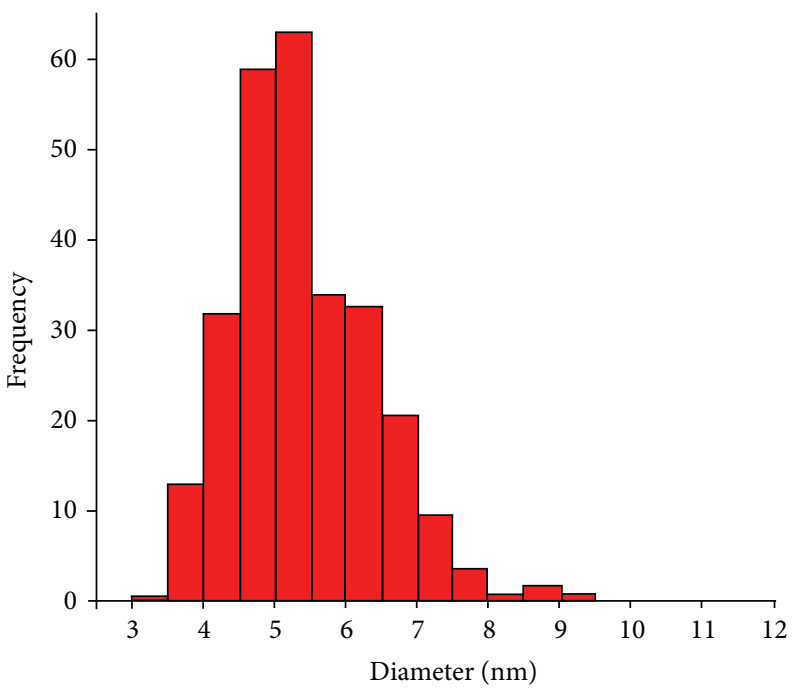

(c)

Figure 5: (a) Micrography of Fe nanoparticles deposited on Si substrate. (b) A magnified micrography of a Fe nanoparticle. (c) Histogram corresponding to deposited Fe nanoparticles obtained from TEM analysis.

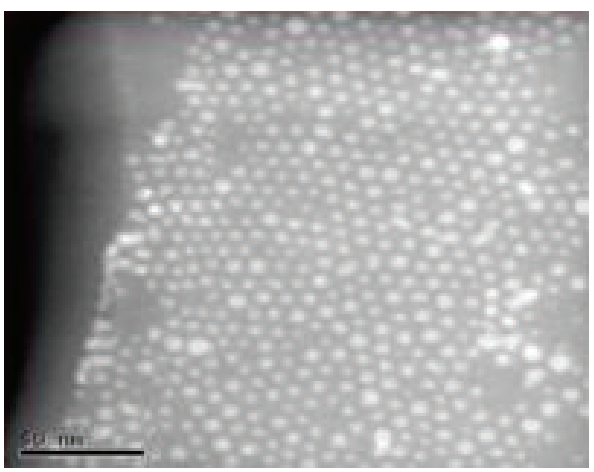

(a)

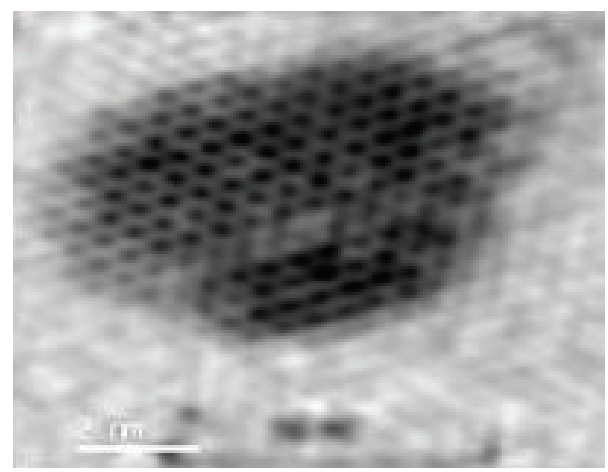

(b)

FIGURE 6: TEM images of Fe nanoparticles coated with oleic acid deposited by IGC.

the effectiveness of inert gas condensation technique to obtain nanoparticles of predetermined size.

Figure 6 shows TEM images of Fe nanoparticles coated with oleic acid deposited by IGC. We can see that particles show high uniformity in a nearly spherical shape with the average diameter of $5 \mathrm{~nm}$. After surface modification, the nanoparticles maintained their original spherical shapes without any deformation or coalescence due to particles 


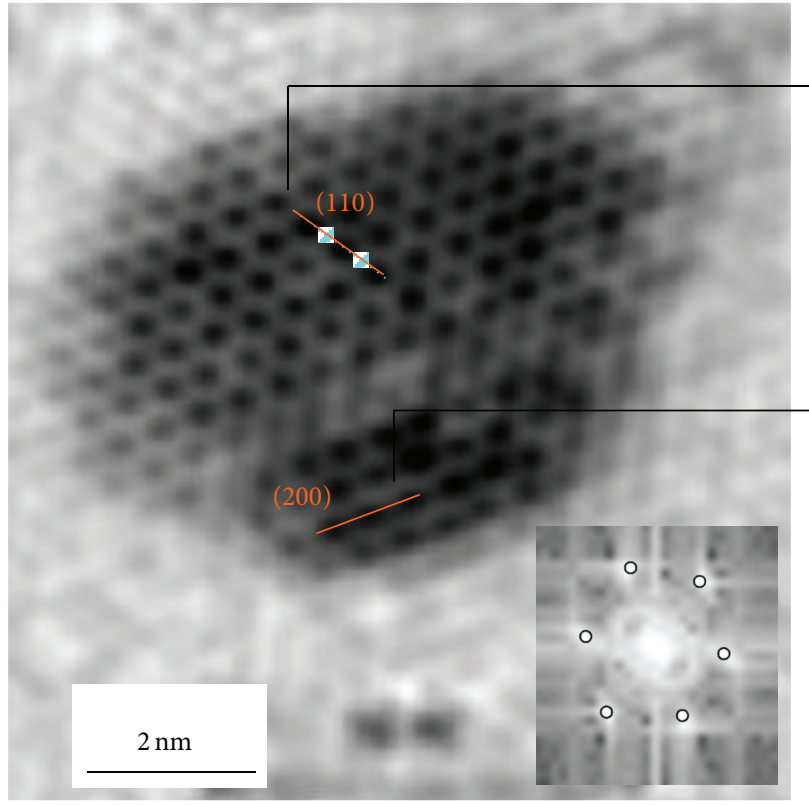

(a)
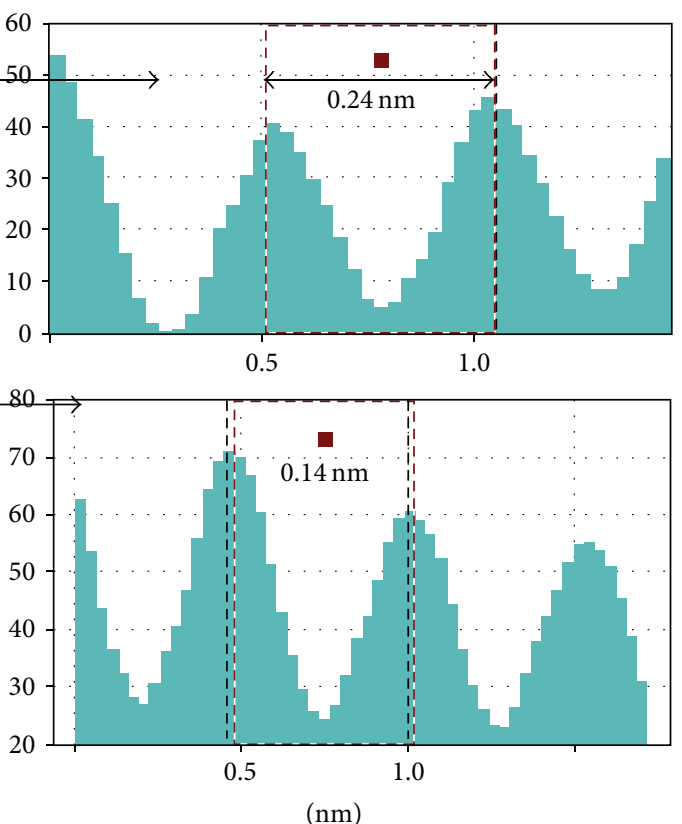

(b)

FIGURE 7: Iron nanoparticles coated with oleic acid obtained in the experiments. The crystallographic distances found by the nanoparticle profile (right) correspond to (100) and (200) planes of cubic Fe (metal).

aggregation and are self-organized in a hexagonal arrangement, confirming that nanoparticle surface has been stabilized. Oleic acid is widely used in ferrite nanoparticle synthesis because it can form dense protective monolayers; producing therefore, highly uniform in shape and monodisperse nanoparticles.

Figure 7 shows the iron nanoparticles obtained in the experiments; the indexing was performed by Carine 3.1 software. The crystallographic distances found by the nanoparticle profile (right) correspond to (100) and (200) planes of cubic Fe (metal), according to the crystallographic chart with reference code 00-006-0696. Phases corresponding to oxides or other materials based on iron were not evidenced; this proves the efficiency of the stabilization method used in this work, whose distribution size, shape, and oxidation were controlled.

Figure 8 shows the background subtracted energy loss spectra for the Fe nanoparticles with and without oleic acid. The label $\mathrm{K}$ indicates the energetic position of the oxygen $\mathrm{K} 1$ selector binding energy. L2 and L3 define the $2 \mathrm{p} 1 / 2$ and $2 \mathrm{p} 3 / 2$ binding energy of iron, respectively [24].

The slight increase of intensity that can be seen at energies above the L3 edge is caused by electron scattering processes. The shift indicates the presence of metallic Fe for the oleic acid capped nanoparticles $[25,26]$.

\section{Conclusions}

From previous results we can say that monodispersed $\mathrm{Fe}$ nanoparticles were obtained. The inert gas condensation technique allows us to prepare in situ oleic acid capped $\mathrm{Fe}$

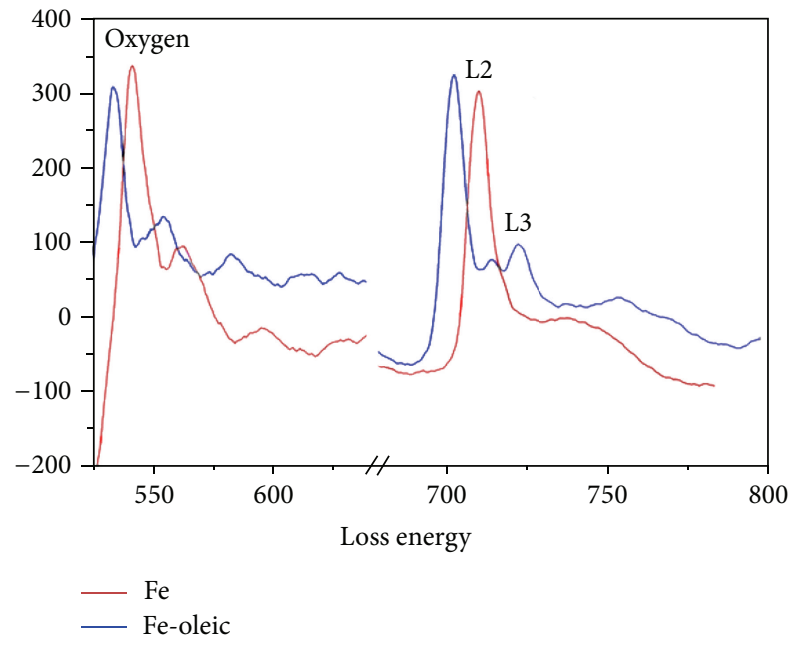

Figure 8: The background subtracted energy loss spectra for the Fe nanoparticles with and without oleic acid.

nanoparticles with a high resolved distribution size. Due to the oleic acid stabilization, iron nanoparticles were selforganized in hexagonal arrays. This result opens a path for some possible applications: catalysis, chemical, biomedical, electronic, biological, and so forth. Due the nature of this kind of applications it is essential to count with a high control grade for the particle size. We were able to obtain Fe nanoparticles highly stabilized with the oleic acid molecule with a focalized size distribution. Phases corresponding to oxides or other 
materials based on iron were not observed, demonstrating the efficiency of the stabilization method used in this work.

\section{Conflict of Interests}

The authors declare that there is no conflict of interests regarding the publication of this paper.

\section{Acknowledgment}

This work was economically supported by CONACYT Mexico.

\section{References}

[1] A. S. Edelstein and R. C. Cammarata, Nanoparticle: Synthesis, Properties and Applications, Institute of Physics Publishing, Bristol, UK, 1996.

[2] G. Schmid, Nanoscale Materials in Chemistry, Wiley, New York, NY, USA, 2001.

[3] R. J. Puddephat, The Chemistry of Gold, Elsevier, Amsterdam, The Netherlands, 1978.

[4] C. M. Niemeyer, "Nanoparticles, proteins, and nucleic acids: biotechnology meets materials science," Angewandte ChemieInternational Edition, vol. 40, no. 22, pp. 4128-4158, 2001.

[5] L. Olofsson, T. Rindzevicius, I. Pfeiffer, M. Käll, and F. Höök, "Surface-based gold-nanoparticle sensor for specific and quantitative DNA hybridization detection," Langmuir, vol. 19, no. 24, pp. 10414-10419, 2003.

[6] T. Ogawa, K. Kobayashi, G. Masuda, T. Takase, and S. Maeda, "Electronic conductive characteristics of devices fabricated with 1,10-decanedithiol and gold nanoparticles between $1-\mu \mathrm{m}$ electrode gaps," Thin Solid Films, vol. 393, no. 1-2, pp. 374-378, 2001.

[7] S.-J. Park, A. A. Lazarides, C. A. Mirkin, and R. L. Letsinger, "Directed assembly of periodic materials from protein and oligonucleotide-modified nanoparticle building blocks," Angewandte Chemie International Edition, vol. 40, pp. 2909-2912, 2001.

[8] K. Kimura, S. Sato, and H. Yao, "Particle crystals of surface modified gold nanoparticles grown from water," Chemistry Letters, no. 4, pp. 372-373, 2001.

[9] D. H. Brown and W. E. Smith, "The chemistry of the gold drugs used in the treatment of rheumatoid arthritis," Chemical Society Reviews, vol. 9, no. 2, pp. 217-240, 1980.

[10] B. H. Schneider, E. L. Dickinson, M. D. Vach, J. V. Hoijer, and L. V. Howard, "Optical chip immunoassay for hCG in human whole blood," Biosensors and Bioelectronics, vol. 15, no. 11-12, pp. 597-604, 2000.

[11] M. Zheng, F. Davidson, and X. Huang, "Ethylene glycol monolayer protected nanoparticles for eliminating nonspecific binding with biological molecules," Journal of the American Chemical Society, vol. 125, no. 26, pp. 7790-7791, 2003.

[12] B. Nolting, J. J. Yu, G. Y. Liu, S. J. Cho, S. Kauzlarich, and J. Gervay-Hague, "Synthesis of gold glyconanoparticles and biological evaluation of recombinant Gp120 interactions," Langmuir, vol. 19, no. 16, pp. 6465-6473, 2003.

[13] C. Zhang, Z. Zhang, B. Yu, J. Shi, and X. Zhang, "Application of the biological conjugate between antibody and colloid $\mathrm{Au}$ nanoparticles as analyte to inductively coupled plasma mass spectrometry," Analytical Chemistry, vol. 74, no. 1, pp. 96-99, 2002.

[14] S. Bharathi, "Sol-gel-derived nanocrystalline gold-silicate composite biosensor," Analytical Communications, vol. 35, no. 1, pp. 29-31, 1998.

[15] J. H. Park, Y. T. Lim, O. O. Park, and Y. C. Kim, "Enhancement of photostability in blue-light-emitting polymers doped with gold nanoparticles," Macromolecular Rapid Communications, vol. 24, no. 4, pp. 331-334, 2003.

[16] A.-H. Lu, E. L. Salabas, and F. Schüth, "Magnetic nanoparticles: synthesis, protection, functionalization, and application," Angewandte Chemie-International Edition, vol. 46, no. 8, pp. 1222 1244, 2007.

[17] Mantis Deposition Limited, Oxford, UK, http://www.mantisdeposition.com.

[18] R. W. J. Scott, A. K. Datye, and R. M. Crooks, "Bimetallic palladium-platinum dendrimer-encapsulated catalysts," Journal of the American Chemical Society, vol. 125, no. 13, pp. 37083709, 2003.

[19] E. Pérez-Tijerina, M. Gracia Pinilla, S. Mejía-Rosales, U. Ortiz-Méndez, A. Torres, and M. José-Yacamán, "Highly sizecontrolled synthesis of $\mathrm{Au} / \mathrm{Pd}$ nanoparticles by inert-gas condensation," Faraday Discussions, vol. 138, pp. 353-362, 2008.

[20] K. Sattler, J. Mühlbach, and E. Recknagel, "Generation of metal clusters containing from 2 to 500 atoms," Physical Review Letters, vol. 45, no. 10, pp. 821-824, 1980.

[21] D. Reinhard, B. D. Hall, D. Ugarte, and R. Monot, "Sizeindependent fcc-to-icosahedral structural transition in unsupported silver clusters: an electron diffraction study of clusters produced by inert-gas aggregation," Physical Review BCondensed Matter and Materials Physics, vol. 55, no. 12, pp. 7868-7881, 1997.

[22] H. Haberland, Z. Insepov, and M. Moseler, "Moleculardynamics simulation of thin-film growth by energetic cluster impact," Physical Review B, vol. 51, no. 16, pp. 11061-11067, 1995.

[23] O. Rattunde, M. Moseler, A. Häfele, J. Kraft, D. Rieser, and H. Haberland, "Surface smoothing by energetic cluster impact," Journal of Applied Physics, vol. 90, no. 7, pp. 3226-3231, 2001.

[24] T. P. Huelser, H. Wiggers, P. Ifeacho, O. Dmitrieva, G. Dumpich, and A. Lorke, "Morphology, structure and electrical properties of iron nanochains," Nanotechnology, vol. 17, no. 13, article 5, pp. 3111-3115, 2006.

[25] R. D. Leapman, L. A. Grunes, and P. L. Fejes, "Study of the $\mathrm{L}_{23}$ edges in the $3 d$ transition metals and their oxides by electronenergy-loss spectroscopy with comparisons to theory," Physical Review B, vol. 26, no. 2, pp. 614-635, 1982.

[26] Y. Jin, H. Xu, and A. K. Datye, "Electron Energy Loss Spectroscopy (EELS) of iron Fischer-Tropsch catalysts," Microscopy and Microanalysis, vol. 12, no. 2, pp. 124-134, 2006. 

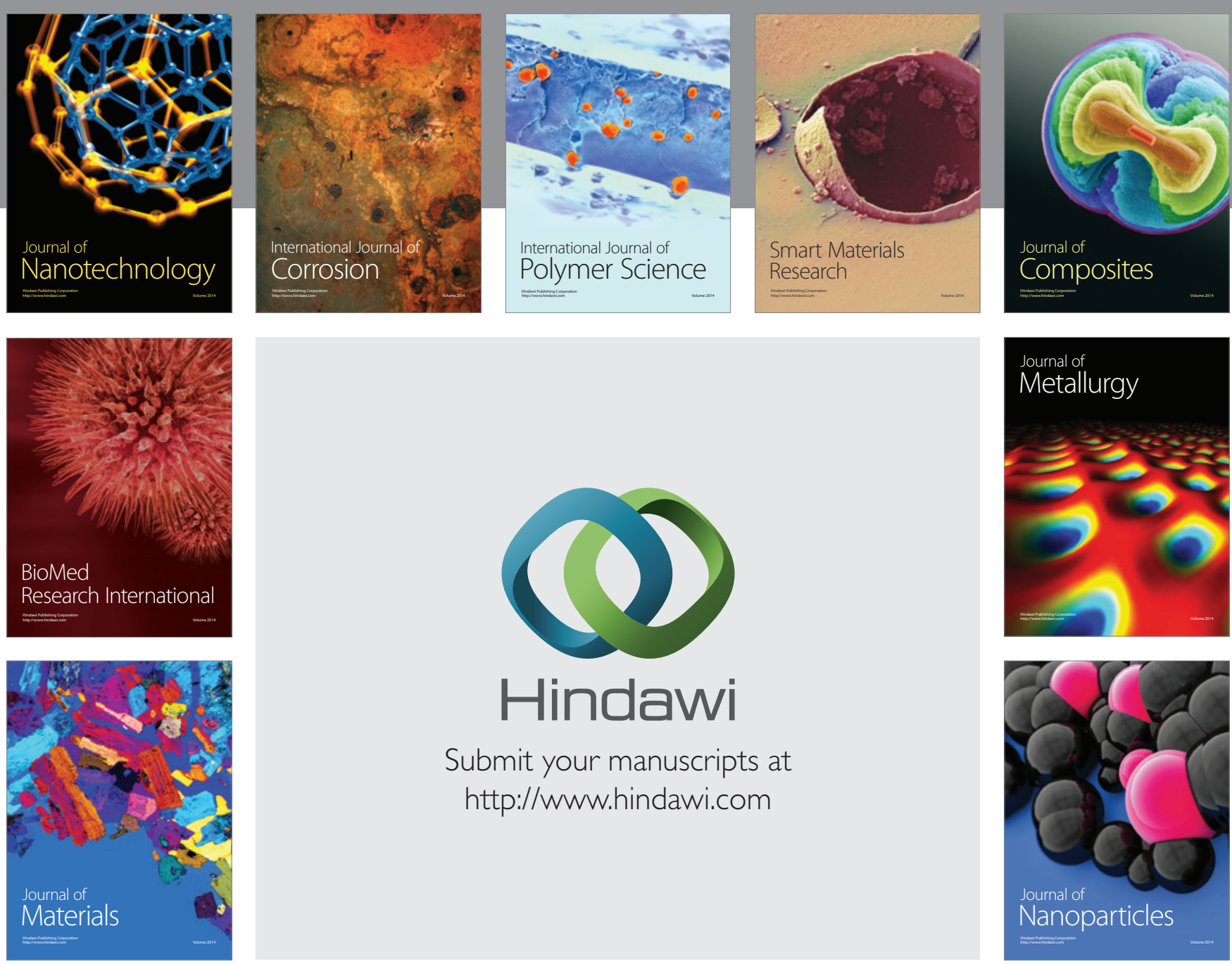

Submit your manuscripts at http://www.hindawi.com
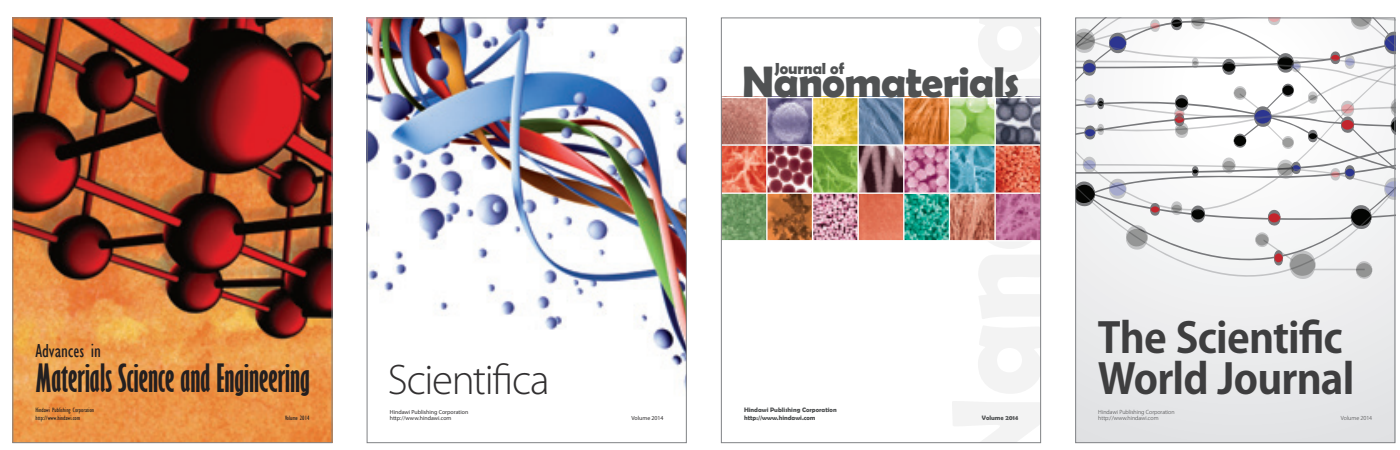

\section{The Scientific World Journal}
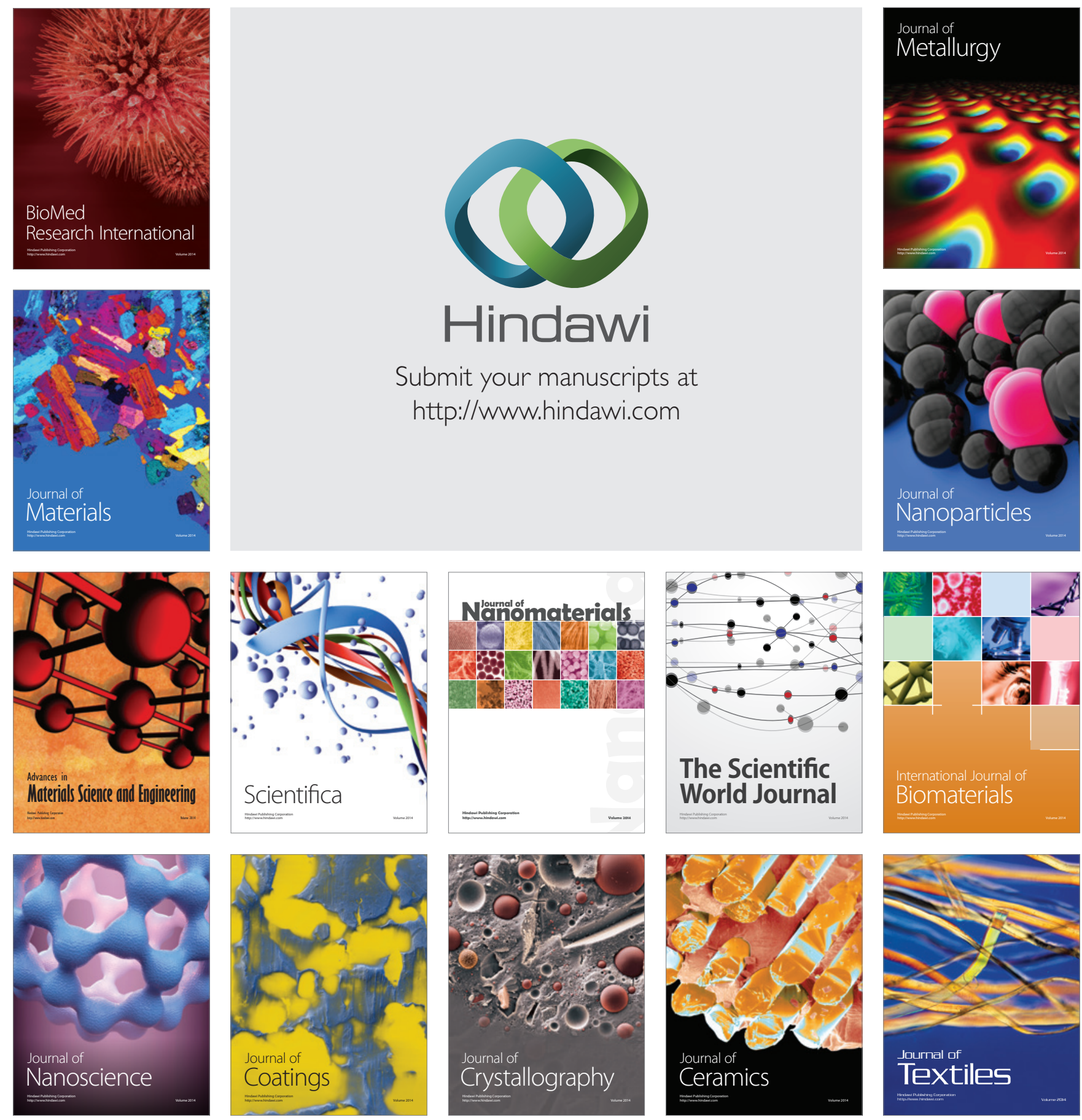